\title{
GALOISIAN APPROACH FOR A STURM-LIOUVILLE PROBLEM ON THE INFINITE INTERVAL*
}

\author{
DAVID BLÁZQUEZ-SANZ† AND KAZUYUKI YAGASAKI†
}

\begin{abstract}
We study a Sturm-Liouville type eigenvalue problem for second-order differential equations on the infinite interval $(-\infty, \infty)$. Here the eigenfunctions are nonzero solutions exponentially decaying at infinity. We prove that at any discrete eigenvalue the differential equations are integrable in the setting of differential Galois theory under general assumptions. Our result is illustrated with three examples for a stationary Schrödinger equation having a generalized Hulthén potential; a linear stability equation for a traveling front in the Allen-Cahn equation; and an eigenvalue problem related to the Lamé equation.
\end{abstract}

Key words. Sturm-Liouville problem, differential Galois theory, integrability, monodromy group.

AMS subject classifications. 34B09, 34B24, 35B35, 81Q05.

1. Introduction. We study a Sturm-Liouville type problem for second-order differential equations of the form

$$
\frac{\mathrm{d}^{2} \psi}{\mathrm{d} x^{2}}+\mu(x) \frac{\mathrm{d} \psi}{\mathrm{d} x}+\nu(x) \psi=\lambda \rho(x) \psi, \quad \lambda \in \mathbb{C},
$$

on the infinite interval $(-\infty, \infty)$ with boundary conditions

$$
\lim _{x \rightarrow \pm \infty} \psi(x)=0
$$

where $\mu, \nu, \rho: \mathbb{R} \rightarrow \mathbb{R}$ are analytic functions. If the boundary value problem $(1,2)$ has a nonzero solution, then the value of $\lambda$ is called an eigenvalue and the nonzero solution $\psi(x)$, which is easily shown to decay exponentially at infinity, is called the associated eigenfunction. See $[4,30]$ and references therein for the history and general results on the Sturm-Liouville problem. This type of equation arises in many mathematical and physical applications including stationary Schrödinger equations [17] and spectral stability of pulses and fronts in partial differential equations (PDEs) [22].

In general, it is difficult to solve the eigenvalue problem $(1,2)$ analytically, and explicit solutions are obtained only in special cases. For stationary Schrödinger equations, in which $\mu(x) \equiv 0$ and $\rho(x) \equiv 1$ in (1), Acosta-Humánez et al. [2] recently studied the eigenvalue problem by means of differential Galois theory [14, 25]. Here the differential Galois theory is an extended version of the classical Galois theory, which treats the solvability of algebraic equations, for differential equations and deals with the problem of integrability by quadratures for them. They computed such values of $\lambda$ as equation (1) has a solvable differential Galois group, for many examples, and showed for some of them that the differential Galois group is solvable if $\lambda$ is an eigenvalue (see also [1]). In this paper, we show that this statement holds for (1) with

\footnotetext{
${ }^{*}$ Received May 13, 2012; accepted for publication November 29, 2012.

†Escuela de Matemáticas, Universidad Sergio Arboleda, Bogotá, Colombia. Present address: Universidad Nacional de Colombia - Sede Medellín, Calle 69 No. 63 - 20, Medellín - Antioquia Colombia (dblazquezs@unal.edu.co).

$\ddagger$ Mathematics Division, Department of Information Engineering, Niigata University, Niigata 9502181, Japan. Present address: Geometric and Algebraic Analysis Group, Department of Mathematics, Hiroshima University, Higashi-Hiroshima 739-8521, Japan (yagasaki@hiroshima-u.ac.jp).
} 


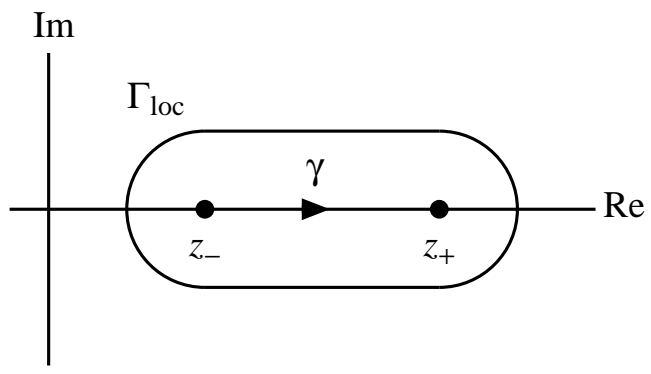

FIG. 1. Assumption (A1) and a simply connected neighborhood $\Gamma_{\text {loc }}$.

$\mu(x) \not \equiv 0$ and $\rho(x) \not \equiv 1$ under general assumptions. We now state our main results precisely.

We first make the following assumptions:

(A1) Let $I \subset \mathbb{R}$ be an open interval. There exist an analytic function $f: I \rightarrow \mathbb{R}$ and two points $z_{ \pm} \in I$ such that $f\left(z_{ \pm}\right)=0, f^{\prime}\left(z_{ \pm}\right) \neq 0$ and

$$
\mu(x)=g(\gamma(x)), \quad \nu(x)=h(\gamma(x)), \quad \rho(x)=k(\gamma(x)),
$$

where the prime represents differentiation with respect to $x ; \gamma(x)$ is a heteroclinic solution in

$$
\frac{\mathrm{d} z}{\mathrm{~d} x}=f(z), \quad z \in \mathbb{R}
$$

connecting the equilibria $z=z_{ \pm}$such that $\lim _{x \rightarrow \pm \infty} \gamma(x)=z_{ \pm}$; and $g(z), h(z), k(z)$ are meromorphic functions in an open set $U$ containing $\{\gamma(x) \mid x \in \mathbb{R}\} \cup\left\{z_{ \pm}\right\}$in $\mathbb{C}$. See Fig. 1

(A2) The functions $g(z), h(z), k(z)$ are holomorphic at $z=z_{ \pm}$.

We easily see that $\mu(x), \nu(x)$ and $\rho(x)$, respectively, converge exponentially to finite values

$$
\mu_{ \pm}=g\left(z_{ \pm}\right), \quad \nu_{ \pm}=h\left(z_{ \pm}\right) \quad \text { and } \quad \rho_{ \pm}=k\left(z_{ \pm}\right)
$$

as $x \rightarrow \pm \infty$, since $f^{\prime}\left(z_{ \pm}\right) \neq 0$. We also have $f^{\prime}\left(z_{+}\right)<0$ and $f^{\prime}\left(z_{-}\right)>0$ since $z=z_{+}$ and $z=z_{-}$must be a sink and source, respectively, in (3).

Under the transformation $z=\gamma(x)$, equation (1) is written as

$$
\frac{\mathrm{d}^{2} \psi}{\mathrm{d} z^{2}}+\frac{g(z)+f^{\prime}(z)}{f(z)} \frac{\mathrm{d} \psi}{\mathrm{d} z}+\frac{h(z)-\lambda k(z)}{f(z)^{2}} \psi=0,
$$

which is regarded as a complex differential equation with meromorphic coefficients. Generally, a singular point in linear differential equations with meromorphic coefficients is called regular if the growth of solutions along any ray approaching the singular point is bounded by a meromorphic function; otherwise it is called irregular. It is well known for second-order differential equations of the form (4) that a singular point $z_{0}$ is regular if the coefficients of $\mathrm{d} \psi / \mathrm{d} z$ and $\psi$ are $O\left(\left(z-z_{0}\right)^{-1}\right)$ and $O\left(\left(z-z_{0}\right)^{-2}\right)$, respectively. See, e.g., $[13,28]$ for more details on this statement. Since $f^{\prime}\left(z_{ \pm}\right) \neq 0$ and $g(z), h(z), k(z)$ are holomorphic at $z_{ \pm}$by assumptions (A1) and (A2), we see that the singular points $z=z_{ \pm}$are regular in (4). 
Let $\Gamma_{\text {loc }}$ be a simply connected neighborhood of $\{\gamma(x) \mid x \in \mathbb{R}\} \cup\{0,1\}$ in $\mathbb{C}$ (see Fig. 1). We prove the following theorem.

Theorem 1.1. Suppose that $z=z_{ \pm}$are the only singularities of (4) in $\Gamma_{\text {loc }}$ and that the eigenvalue $\lambda$ is located in a complex region $R$ given by

$$
\mu_{ \pm}^{2}\left(\lambda_{\mathrm{R}} \rho_{ \pm}-\nu_{ \pm}\right)+\lambda_{\mathrm{I}}^{2} \rho_{ \pm}^{2}>0 \quad \text { or } \quad \mu_{ \pm}^{2}<4\left(\lambda_{\mathrm{R}} \rho_{ \pm}-\nu_{ \pm}\right),
$$

where $\lambda_{\mathrm{R}}=\operatorname{Re}(\lambda)$ and $\lambda_{\mathrm{I}}=\operatorname{Im}(\lambda)$. If the boundary value problem $(1,2)$ has a nonzero solution, then the restriction of (4) onto $\Gamma_{\text {loc }}$ has a triangularizable differential Galois group.

Roughly speaking, this theorem means that if $\lambda$ is an eigenvalue satisfying (5), then equation (1) is integrable in the setting of the differential Galois theory. We will also see that an eigenvalue is not discrete if it does not satisfy (5) (see Remark 3.5). In some case all eigenvalues of the problem $(1,2)$ satisfy $(5)$. We also obtain the following result as a corollary of Theorem 1.1.

THEOREM 1.2. Suppose that the two points $z=z_{ \pm}$are the only singularities of (4) in $\Gamma_{\text {loc }}$ and one of the following conditions is satisfied:

(i) $\mu_{+} \leq 0 \leq \mu_{-}$(in particular, $\left.\mu_{ \pm}=0\right)$;

(ii) $\mu_{+}>0, \mu_{-} \geq 0$ and condition (5-) holds;

(iii) $\mu_{+} \leq 0, \mu_{-}<0$ and condition ( $\left.5_{+}\right)$holds.

Then the conclusion of Theorem 1.1 holds.

Here we have said that condition $\left(5_{+}\right)$(resp. $\left(5_{-}\right)$) holds if condition (5) holds for the sign "+" (resp. "-"). To prove the main theorems, we analyze (4) using the differential Galois theory. Similar techniques were used to study bifurcations of homoclinic orbits in [6] very recently and horseshoe dynamics in [20,29] much earlier. Fauvet et al. [9] also studied an eigenvalue problem for a special non-Fuchsian secondorder differential equation called the prolate spheroidal wave equation [27] on a finite interval, using the differential Galois theory. They analyzed the Stokes phenomenon and clarified a relation between solutions of the eigenvalue problem and the differential Galois group.

The rest of the paper is organized as follows. We provide necessary information on the differential Galois theory in Sec. 2 and give proofs of Theorems 1.1 and 1.2 in Sec. 3. In Sec. 4, our result is illustrated with three examples for a stationary Schrödinger equation having a generalized Hulthén potential [12]; a linear stability equation for a traveling front in the Allen-Cahn equation [3]; and an eigenvalue problem related to the Lamé equation [28]. The third example may be a little artificial but it demonstrates the ability of our result, which is not just an application of hypergeometric functions.

2. Differential Galois theory. We briefly review a part of the differential Galois theory which is often referred to as the Picard-Vessiot theory and gives a complete framework about the integrability by quadratures of linear differential equations with variable coefficients.

2.1. Picard-Vessiot extensions and differential Galois groups. Consider a system of abstract differential equations

$$
\partial y=A y, \quad A \in \operatorname{gl}(n, \mathbb{K}),
$$

where $\partial$ represents a derivation, which is an additive endomorphism satisfying the Leibniz rule; $\mathbb{K}$ is a differential field, i.e., a field endowed with the derivation $\partial$; and 
$\operatorname{gl}(n, \mathbb{K})$ denotes the ring of $n \times n$ matrices with entries in $\mathbb{K}$. The set $\mathrm{C}_{\mathbb{K}}$ of elements of $\mathbb{K}$ for which $\partial$ vanishes is a subfield of $\mathbb{K}$ and called the field of constants for $\mathbb{K}$. In our application in this paper, the differential field $\mathbb{K}$ is the field of meromorphic functions on a Riemann surface, so that the field of constants becomes that of complex numbers, $\mathbb{C}$. A differential field extension $\mathbb{L} \supset \mathbb{K}$ is a field extension such that $\mathbb{L}$ is also a differential field and the derivations on $\mathbb{L}$ and $\mathbb{K}$ coincide on $\mathbb{K}$.

Definition 2.1. A Picard-Vessiot extension for (6) is a differential field extension $\mathbb{L} \supset \mathbb{K}$ satisfying the following:

(PV1) There is a fundamental matrix $\Phi$ of (6) with coefficients in $\mathbb{L}$.

(PV2) The field $\mathbb{L}$ is spanned by $\mathbb{K}$ and entries of the fundamental matrix $\Phi$.

(PV3) The field of constants for $\mathbb{L}$ coincides with that for $\mathbb{K}$.

The system (6) admits a Picard-Vessiot extension which is unique up to isomorphism. If $\mathbb{K}$ is the field of meromorphic functions on a Riemann surface, then we have a fundamental matrix in some field of convergent Laurent series, and get the Picard-Vessiot extension by adding convergent Laurent series to $\mathbb{K}$.

We now fix a Picard-Vessiot extension $\mathbb{L} \supset \mathbb{K}$ and fundamental matrix $\Phi$ with coefficients in $\mathbb{L}$ for (6). The constant field of $\mathbb{L}$ coincides with that of $\mathbb{K}$, which is denoted by C. Let $\sigma$ be a $\mathbb{K}$-automorphism of $\mathbb{L}$, i.e., a field automorphism of $\mathbb{L}$ that commutes with the derivation of $\mathbb{L}$ and leaves $\mathbb{K}$ pointwise fixed. Obviously, $\sigma(\Phi)$ is also a fundamental matrix of (6) and consequently there is a matrix $m_{\sigma}$ with constant entries such that $\sigma(\Phi)=\Phi m_{\sigma}$. This relation gives a faithful representation of the group of $\mathbb{K}$-automorphisms of $\mathbb{L}$ on the general linear group as

$$
\text { gal: } \operatorname{Aut}_{\mathbb{K}}(\mathbb{L}) \rightarrow \mathrm{GL}(n, \mathrm{C}), \quad \sigma \mapsto m_{\sigma},
$$

where $\operatorname{Aut}_{\mathbb{K}}(\mathbb{L})$ is the set of $\mathbb{K}$-automorphisms of $\mathbb{L}$, and $\operatorname{GL}(n, C)$ is the group of $n \times n$ invertible matrices with entries in C. The image of the representation "gal" is a linear algebraic subgroup of $\operatorname{GL}(n, \mathrm{C})$, which is called the differential Galois group of (6) and denoted by $\operatorname{Gal}(\mathbb{L} / \mathbb{K})$. This representation is not unique and depends on the choice of the fundamental matrix $\Phi$, but a different fundamental matrix only gives rise to a conjugated representation. Thus, the differential Galois group is unique up to conjugation as an algebraic subgroup of the general linear group over C.

Definition 2.2. A differential field extension $\mathbb{L} \supset \mathbb{K}$ is called

(i) an integral extension if there exists $a \in \mathbb{L}$ such that $a^{\prime} \in \mathbb{K}$ and $\mathbb{L}=\mathbb{K}(a)$, where $\mathbb{K}(a)$ is the smallest extension of $\mathbb{K}$ containing $a$;

(ii) an exponential extension if there exists $a \in \mathbb{L}$ such that $a^{\prime} / a \in \mathbb{K}$ and $\mathbb{L}=\mathbb{K}(a)$;

(iii) an algebraic extension if there exists $a \in \mathbb{L}$ such that it is algebraic over $\mathbb{K}$ and $\mathbb{L}=\mathbb{K}(a)$.

Here a prime represents differentiation, i.e., $a^{\prime}=\partial a$, as usual.

Definition 2.3. A differential field extension $\mathbb{L} \supset \mathbb{K}$ is called a Liouvillian extension if it can be decomposed as a tower of extensions,

$$
\mathbb{L}=\mathbb{K}_{n} \supset \ldots \supset \mathbb{K}_{1} \supset \mathbb{K}_{0}=\mathbb{K},
$$

such that each extension $\mathbb{K}_{i+1} \supset \mathbb{K}_{i}$ is either integral, exponential or algebraic. It is called strictly Liouvillian if in the tower only integral and exponential extensions appear. 
In general, an algebraic group $G \subset \mathrm{GL}(n, \mathrm{C})$ contains a unique maximal connected algebraic subgroup $G^{0}$ called the connected component of the identity or connected identity component. The connected identity component $G^{0} \subset G$ is a normal algebraic subgroup and the smallest subgroup of finite index, i.e., the quotient group $G / G^{0}$ is finite. By the Lie-Kolchin Theorem [14, 25], a connected solvable linear algebraic group is triangularizable. Here a subgroup of $\operatorname{GL}(n, \mathrm{C})$ is said to be triangularizable if it is conjugated to a subgroup of the group of upper triangular matrices. The following theorem relates the solvability and triangularizability of the differential Galois group with the (strictly) Liouvillian Picard-Vessiot extension (see [14, 25] and [5] for the proofs of the first and second parts, respectively).

TheOREM 2.4. Let $\mathbb{L} \supset \mathbb{K}$ be a Picard-Vessiot extension of (6).

(i) The connected identity component of the differential Galois group $\operatorname{Gal}(\mathbb{K} / \mathbb{L})$ is solvable if and only if $\mathbb{L} \supset \mathbb{K}$ is a Liouvillian extension.

(ii) If the differential Galois group $\operatorname{Gal}(\mathbb{K} / \mathbb{L})$ is triangularizable, then $\mathbb{L} \supset \mathbb{K}$ is a strictly Liouvillian extension.

2.2. Monodromy groups and Fuchsian equations. Let $\mathbb{K}$ be the field of meromorphic functions on a Riemann surface $\Gamma$ and let $z_{0} \in \Gamma$ be a nonsingular point in (6). We prolong the fundamental matrix $\Phi(z)$ analytically along any loop $\ell$ based at $z_{0}$ and containing no singular point, and obtain another fundamental matrix $\ell * \Phi(z)$. So there exists a constant nonsingular matrix $M_{\ell}$ such that

$$
\ell * \Phi(z)=\Phi(z) M_{\ell}
$$

We call $M_{\ell}$ the monodromy matrix for $\ell$. The set of singularities in (6), which is denoted by $S$, is a discrete subset of $\Gamma$. Let $\pi_{1}\left(\Gamma \backslash S, z_{0}\right)$ be the fundamental group of homotopy classes of loops based at $z_{0}$. The monodromy matrix $M_{\ell}$ depends on the homotopy class $[\ell]$ of the loop $\ell$, and it is also denoted by $M_{[\ell]}$. We have a representation

$$
\text { mon: } \pi_{1}\left(\Gamma \backslash S, z_{0}\right) \rightarrow \mathrm{GL}(n, \mathbb{C}), \quad[\ell] \mapsto M_{[\ell]}
$$

The image of "mon" is called the monodromy group of (6). As in the differential Galois group, the representation "mon" depends on the choice of the fundamental matrix, but the monodromy group is defined as a group of matrices up to conjugation. In general, monodromy transformations define automorphisms of the corresponding Picard-Vessiot extension.

Recall that equation (6) is said to be Fuchsian if all singularities are regular. For Fuchsian equations we have the following result (see, e.g., Theorem 5.8 in [25] for the proof).

TheOrem 2.5 (Schlessinger). Assume that equation (6) is Fuchsian. Then the differential Galois group of (6) is the Zariski closure of the monodromy group.

Since the group of triangular matrices is algebraic, the Zariski closure of a triangularizable group is triangularizable. Noting this fact, we obtain the following result immediately from Theorem 2.5.

Corollary 2.6. Assume that equation (6) is Fuchsian. Then the monodromy group is triangularizable if and only if the differential Galois group is triangularizable. 
3. Proofs of Theorems 1.1 and 1.2. We first consider general second-order differential equations of the form

$$
\frac{\mathrm{d}^{2} u}{\mathrm{~d} z^{2}}+\frac{f_{1}(z)}{z} \frac{\mathrm{d} u}{\mathrm{~d} z}+\frac{f_{2}(z)}{z^{2}} u=0
$$

on $\mathbb{C}$, where $f_{1}(z)$ and $f_{2}(z)$ are holomorphic at $z=0$. Equation (7) is rewritten in the form (6) with

$$
y=\left(\begin{array}{c}
u \\
u^{\prime}
\end{array}\right) \quad \text { and } \quad A(z)=\left(\begin{array}{cc}
0 & 1 \\
-f_{1}(z) / z & f_{2}(z) / z^{2}
\end{array}\right)
$$

where a prime represents differentiation with respect to $z$.

The origin $z=0$ is a regular singularity in (7). Let $\rho_{1}, \rho_{2}$ be the local exponents of (7) at $z=0$, i.e., roots of of the indicial equation

$$
s(s-1)+f_{1}(0) s+f_{2}(0)=0 .
$$

The following result is classical and well known (see, e.g., [13, 28]).

Lemma 3.1. Around $z=0$, equation (7) has two independent solutions, $u_{j}(z)$, $j=1,2$, of the following forms:

(i) If $\rho_{1}-\rho_{2}$ is not an integer, then

$$
u_{1}(z)=z^{\rho_{1}} v_{1}(z), \quad u_{2}(z)=z^{\rho_{2}} v_{2}(z)
$$

(ii) if $\rho_{1}-\rho_{2}$ is a nonnegative integer, then they are given by (9) or

$$
u_{1}(z)=z^{\rho_{1}} v_{1}(z), \quad u_{2}(z)=z^{\rho_{2}} v_{2}(z)+u_{1}(z) \log z .
$$

Here $v_{1}(z), v_{2}(z)$ denote some functions which are holomorphic at $z=0$.

We identify (7) with the associated linear system of the form (6) in the context of Sec. 2. Using Lemma 3.1, we obtain the following result (cf. Lemma 4.6 of [6]).

LEMMA 3.2. Suppose that the indicial equation (8) has roots $\rho_{1}, \rho_{2}$ such that $\operatorname{Re}\left(\rho_{2}\right)<0<\operatorname{Re}\left(\rho_{1}\right)$. Then we have the following statements for (7) and the associated linear system of the form (6):

(i) There exists a nonzero solution $\bar{u}(z)$ which is bounded along any ray approaching $z=0$;

(ii) any other independent solution is unbounded along any ray approaching $z=0$;

(iii) the monodromy matrix $M_{\ell}$ has an eigenvalue $e^{2 \pi i \rho_{1}}$ and

$$
\left(\begin{array}{c}
\bar{u}(z) \\
\bar{u}^{\prime}(z)
\end{array}\right)
$$

is the associated eigenvector, where $\ell$ is a sufficiently small loop around $z=0$ in $\mathbb{C}$.

Proof. Parts (i) and (ii) immediately follows from Lemma 3.1. It remains to prove part (iii).

Using Lemma 3.1, we compute the monodromy matrix $M_{\ell}$ for the loop $\ell$ as

$$
M_{\ell}=\left(\begin{array}{cc}
e^{2 \pi i \rho_{1}} & 0 \\
0 & e^{2 \pi i \rho_{2}}
\end{array}\right) \quad \text { or } \quad\left(\begin{array}{cc}
e^{2 \pi i \rho_{1}} & 0 \\
2 \pi i & e^{2 \pi i \rho_{1}}
\end{array}\right)
$$


in the basis $\left\{u_{1}(z), u_{2}(z)\right\}$. Hence, $e^{2 \pi i \rho_{1}}$ is an eigenvalue of $M_{\ell}$ and $u_{1}(z)$ gives the associated eigenvector for both cases. Noting that the bounded solution $\bar{u}(z)$ corresponds to $u_{1}(z)$, we prove part (iii).

Now we are in a position to prove Theorem 1.1.

Proof of Theorem 1.1. Suppose that condition (5) holds. Since

$$
\left(\mu_{ \pm}^{2}+4\left(\lambda_{\mathrm{R}} \rho_{ \pm}-\nu_{ \pm}\right)\right)^{2}-\left(\mu_{ \pm}^{2}-4\left(\lambda_{\mathrm{R}} \rho_{ \pm}-\nu_{ \pm}\right)\right)^{2}=16 \mu_{ \pm}^{2}\left(\lambda_{\mathrm{R}} \rho_{ \pm}-\nu_{ \pm}\right),
$$

we have

$$
\sqrt{\left(\mu_{ \pm}^{2}+4\left(\lambda_{\mathrm{R}} \rho_{ \pm}-\nu_{ \pm}\right)\right)^{2}+16 \lambda_{\mathrm{I}}^{2} \rho_{ \pm}^{2}}>\mu_{ \pm}^{2}-4\left(\lambda_{\mathrm{R}} \rho_{ \pm}-\nu_{ \pm}\right)
$$

and equivalently

$$
\operatorname{Re}\left(\sqrt{\mu_{ \pm}^{2}+4\left(\lambda \rho_{ \pm}-\nu_{ \pm}\right)}\right)>\left|\mu_{ \pm}\right|
$$

Here we have taken a branch of the square root function $\sqrt{z}$ which is positive when $z$ is real and positive, so that

$$
\operatorname{Re} \sqrt{z}=\sqrt{\frac{1}{2}\left(\operatorname{Re} z+\sqrt{(\operatorname{Re} z)^{2}+(\operatorname{Im} z)^{2}}\right)}
$$

for $z \in \mathbb{C}$. Noting that $f\left(z_{ \pm}\right)=0$ and $f^{\prime}\left(z_{ \pm}\right) \neq 0$ by assumption (A1), we write the indicial equations of (4) at $z=z_{ \pm}$as

$$
s(s-1)+\left(a_{ \pm} \mu_{ \pm}+1\right) s+a_{ \pm}^{2}\left(\nu_{ \pm}-\lambda \rho_{ \pm}\right)=s^{2}+a_{ \pm} \mu_{ \pm} s+a_{ \pm}^{2}\left(\nu_{ \pm}-\lambda \rho_{ \pm}\right)=0,
$$

where $a_{ \pm}=1 / f^{\prime}\left(z_{ \pm}\right) \neq 0$. From (10) we easily see that the indicial equation (11) has roots with positive and negative real parts. Hence, it follows from Lemmas 3.1 and 3.2 that equation (4) has only one bounded independent solution of the form

$$
\psi_{ \pm}(z)=\left(z-z_{ \pm}\right)^{\chi \pm} v_{ \pm}(z)
$$

around each of $z=z_{ \pm}$, where $\chi_{ \pm}$represent roots of (11) with positive real parts and $v_{ \pm}(z)$ are holomorphic at $z=z_{ \pm}$. Moreover, by Lemma 3.2(iii), $\psi_{ \pm}(z)$ give eigenvectors of the monodromy matrices $M_{\ell_{ \pm}}$for loops $\ell_{ \pm}$around $z=z_{ \pm}$in $\mathbb{C}$.

Assume that the boundary value problem $(1,2)$ has a nonzero solution $\psi(x)$. Since the solution of (1) must be represented as $\psi(x)=\psi_{ \pm}(\gamma(x))$, we have $\psi_{+}(z)=$ $\psi_{-}(z)$. Hence, the monodromy matrices $M_{\ell_{ \pm}}$have a common eigenvector, so that the monodromy group for (4) is triangularizable. Appealing to Corollary 2.6, we complete the proof.

We turn to the proof of Theorem 1.2. Letting $\psi_{1}=\psi$ and $\psi_{2}=\mathrm{d} \psi / d x$, we rewrite (1) as

$$
\frac{\mathrm{d}}{\mathrm{d} x}\left(\begin{array}{l}
\psi_{1} \\
\psi_{2}
\end{array}\right)=\left(\begin{array}{cc}
0 & 1 \\
\lambda \rho(x)-\nu(x) & -\mu(x)
\end{array}\right)\left(\begin{array}{l}
\psi_{1} \\
\psi_{2}
\end{array}\right) .
$$

The coefficient matrix of (12) exponentially converges to

$$
A_{ \pm}(\lambda)=\left(\begin{array}{cc}
0 & 1 \\
\lambda \rho_{ \pm}-\nu_{ \pm} & -\mu_{ \pm}
\end{array}\right)
$$


as $x \rightarrow \pm \infty$.

Lemma 3.3. Suppose that one of conditions (i)-(iii) in Theorem 1.2 holds and the boundary value problem $(1,2)$ has a nonzero solution. Then condition (5) holds.

Proof. Let $\kappa_{ \pm}$be eigenvalues of the matrices $A_{ \pm}(\lambda)$. By a classical result on linear differential equations (see Sec. 8 and also Problems 29 and 35 in Chapter 3 of [8]), we see that equation (12) has a nonzero solution $\left(\psi_{1}(x), \psi_{2}(x)\right)$ such that

$$
\lim _{x \rightarrow \pm \infty} \psi_{j}(x) e^{-\kappa_{ \pm} x}=c_{j}, \quad j=1,2
$$

for any constants $c_{j}, j=1,2$.

Assume that equation (12) has a nonzero bounded solution. Then for some eigenvalue $\kappa_{ \pm}, e^{\kappa_{ \pm} x}$ must tend to zero as $x \rightarrow \pm \infty$ so that $\operatorname{Re}(\kappa+)<0$ and $\operatorname{Re}\left(\kappa_{-}\right)>0$. This means that a root of the quadratic equation

$$
s^{2}+\mu_{ \pm} s-\left(\lambda \rho_{ \pm}-\nu_{ \pm}\right)=0
$$

has negative and positive real parts for the signs + and - , respectively. Noting that equation (10) means (5) for each sign of \pm , we see that condition $\left(5_{+}\right)$(resp. (5-)) holds if $\mu_{+} \leq 0$ (resp. $\mu_{-} \geq 0$ ). Thus, if one of conditions (i)-(iii) in Theorem 1.2 holds, then condition (5) holds for both signs \pm .

From the proof of Lemma 3.3 we also see that eigenfunctions of the problem $(1,2)$ decay exponentially at infinity.

Proof of Theorem 1.2. Using Lemma 3.3, we obtain Theorem 1.2 as a corollary of Theorem 1.1.

Remark 3.4. Suppose that equation (4) is Fuchsian on the Riemann sphere $\mathbb{P}^{1}$ and has only three singularities at $z=z_{ \pm}$and $z_{*}$, where $z_{*} \in \mathbb{P}^{1}$. Then the surface $\Gamma_{\text {loc }} \backslash\left\{z_{ \pm}\right\}$is homotopic to $\mathbb{P}^{1} \backslash\left\{z_{ \pm}, z_{*}\right\}$, so that they give rise to equivalent monodromy representations. Hence, we see via Theorems 1.1 and 2.5 that equation (4) is integrable by Liouvillian functions on $\mathbb{C}(z)$, which lie in a Liouvillian extension of $\mathbb{C}(z)$ by definition, if the boundary value problem $(1,2)$ has a nonzero solution. This situation happens in examples of Secs. 4.1 and 4.2 .

REMARK 3.5. From the proof of Lemma 3.3 we see that if all eigenvalues of $A_{+}(\lambda)$ have negative real parts and an eigenvalue of $A_{-}(\lambda)$ has a positive real part, or if all eigenvalues of $A_{-}(\lambda)$ have positive real parts and an eigenvalue of $A_{+}(\lambda)$ has a negative real part, then the boundary value problem $(1,2)$ has a nonzero solution. On the other hand, if all eigenvalues of $A_{+}(\lambda)$ have positive real parts or all eigenvalues of $A_{-}(\lambda)$ have negative real parts, then it has no nonzero solution. Hence, $\lambda=\lambda_{\mathrm{R}}+i \lambda_{\mathrm{I}}$ is an eigenvalue of the problem $(1,2)$ if

(i) $\mu_{+}>0, \mu_{-} \geq 0$ and condition (5-) holds but

$$
\mu_{+}^{2}\left(\lambda_{\mathrm{R}} \rho_{+}-\nu_{+}\right)+\lambda_{I}^{2} \leq 0 \quad \text { and } \quad \mu_{+}^{2} \geq 4\left(\lambda_{\mathrm{R}} \rho_{+}-\nu_{+}\right) ;
$$

(ii) $\mu_{+} \leq 0, \mu_{-}<0$ and condition $\left(5_{+}\right)$holds but

$$
\mu_{-}^{2}\left(\lambda_{\mathrm{R}} \rho_{-}-\nu_{-}\right)+\lambda_{I}^{2} \leq 0 \quad \text { and } \quad \mu_{-}^{2} \geq 4\left(\lambda_{\mathrm{R}} \rho_{-}-\nu_{-}\right) ;
$$

(iii) $\mu_{-}<0<\mu_{+}$and condition (14) or (15) holds. 


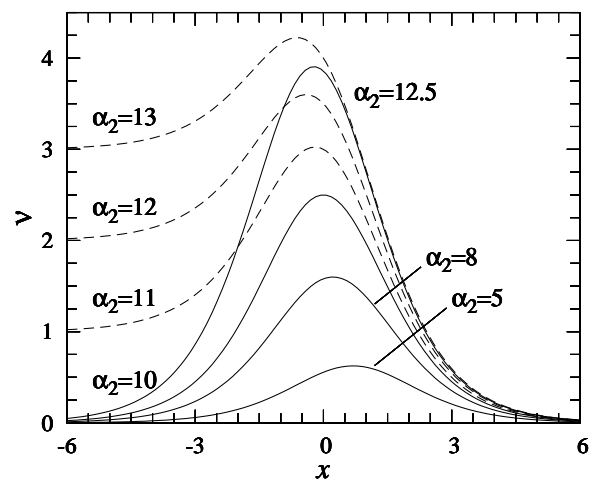

FIG. 2. Shape of the function $\nu(x)$ in (16) for several values of $\alpha_{2}$ when $\alpha_{1}=10 / \alpha_{2}$ or 1 and $\alpha_{3}=10$. Solid and dashed lines represent the cases of $\alpha_{1}=10 / \alpha_{2}$ and 1 , respectively.

Note that these eigenvalues are continuous spectra for the eigenvalue problem $(1,2)$. Moreover, $\lambda$ is not an eigenvalue of the problem $(1,2)$ if

(iv) $\mu_{+} \leq 0$ and condition (14) holds;

(v) $\mu_{-} \geq 0$ and condition (15) holds.

Thus, we can determine all eigenvalues even if neither of conditions (i)-(iii) in Theorem 1.2 holds.

4. Examples. To illustrate the above theory, we give three examples. The first two examples are a Schrödinger equation with a generalized Hulthén potential and a linear stability equation for a traveling front in the Allen-Cahn equation, and can be transformed to the hypergeometric equation. The last one is artificial but transformed not to the hypergeometric equation but to the Lamé equation [28], so that the capability of our result is further demonstrated.

4.1. Schrödinger equation with a generalized Hulthén potential. We first consider a case in which

$$
\mu(x)=0, \quad \nu(x)=\frac{\alpha_{2}}{e^{x}+\alpha_{1}}-\frac{\alpha_{3}}{\left(e^{x}+\alpha_{1}\right)^{2}}, \quad \rho(x)=1,
$$

where $\alpha_{j}, j=1,2,3$, are constants with $\alpha_{j}>0, j=1,2,3$. For (16) equation (1) corresponds to a Schrödinger equation with the generalized Hulthén potential, which is a special case of [31]. A similar but more specific potential was also treated in $[15,22]$. We take

$$
f(z)=z(1-z)
$$

so that equation (3) has two equilibria at $z=0,1$ and a heteroclinic orbit

$$
\gamma(x)=\frac{e^{x}}{e^{x}+1}
$$

from $z=0$ to $z=1$. We easily see that assumptions (A1) and (A2) hold with $z_{-}=0$ and $z_{+}=1$, and

$$
g(z)=0, \quad h(z)=\frac{\alpha_{2}(z-1)}{\left(\alpha_{1}-1\right) z-\alpha_{1}}-\frac{\alpha_{3}(z-1)^{2}}{\left(\left(\alpha_{1}-1\right) z-\alpha_{1}\right)^{2}}, \quad k(z)=1 .
$$


We also have

$$
\mu_{ \pm}=0, \quad \nu_{-}=\frac{\alpha_{2}}{\alpha_{1}}-\frac{\alpha_{3}}{\alpha_{1}^{2}}, \quad \nu_{+}=0, \quad \sup _{x \in \mathbb{R}} \nu(x)=\frac{\alpha_{2}^{2}}{4 \alpha_{3}}, \quad \rho_{ \pm}=1
$$

See Figure 2 for the shape of the function $\nu(x)$ with several values of $\alpha_{2}$ when $\alpha_{1}=$ $10 / \alpha_{2}$ or 1 and $\alpha_{3}=10$.

Equation (4) becomes

$$
\psi^{\prime \prime}+\frac{2 z-1}{z(z-1)} \psi^{\prime}+\frac{h(z)-\lambda}{z^{2}(z-1)^{2}} \psi=0,
$$

which has only regular singularities at $z=0,1, z_{0}$, where

$$
z_{0}= \begin{cases}\alpha_{1} /\left(\alpha_{1}-1\right) & \text { for } \alpha_{1} \neq 1 \\ \infty & \text { for } \alpha_{1}=1\end{cases}
$$

Solutions of (19) are expressed by a Riemann $P$ function $[13,28]$ as

$$
P\left\{\begin{array}{cccc}
0 & 1 & z_{0} & \\
\sigma_{1}^{+} & \sigma_{2}^{+} & \sigma_{3}^{+} & z \\
\sigma_{1}^{-} & \sigma_{2}^{-} & \sigma_{3}^{-} &
\end{array}\right\}
$$

where $\sigma_{1}^{ \pm}, \sigma_{2}^{ \pm}$and $\sigma_{3}^{ \pm}$represent the local exponents of (19) at $z=0,1$ and $z_{0}$, respectively, and are given by

$$
\sigma_{1}^{ \pm}= \pm \sqrt{\lambda-\nu_{-}}, \quad \sigma_{2}^{ \pm}= \pm \sqrt{\lambda}, \quad \sigma_{3}^{ \pm}=\frac{1}{2}\left(1 \pm \frac{1}{\alpha_{1}} \sqrt{\alpha_{1}^{2}+4 \alpha_{3}}\right)
$$

The following result was essentially proved in [16].

Proposition 4.1. Consider a general Fuchsian second-order differential equation having three singularities $z=z_{j}, j=1,2,3$, and a Riemann $P$ function

$$
P\left\{\begin{array}{cccc}
z_{1} & z_{2} & z_{3} & \\
\sigma_{1}^{+} & \sigma_{2}^{+} & \sigma_{3}^{+} & z \\
\sigma_{1}^{-} & \sigma_{2}^{-} & \sigma_{3}^{-} &
\end{array}\right\}
$$

Its monodromy and differential Galois groups are triangularizable if and only if at least one of $\sigma_{1}+\sigma_{2}+\sigma_{3},-\sigma_{1}+\sigma_{2}+\sigma_{3}, \sigma_{1}-\sigma_{2}+\sigma_{3}$ and $\sigma_{1}+\sigma_{2}-\sigma_{3}$ is an odd integer, where $\sigma_{j}=\sigma_{j}^{+}-\sigma_{j}^{-}, j=1,2,3$, denote the exponent differences.

From Proposition 4.1 we see that the monodromy and differential Galois groups for (19) are triangularizable if and only if

$$
\pm 2 \sqrt{\lambda-\nu_{-}} \pm 2 \sqrt{\lambda} \pm \bar{\sigma}_{3}=2 \kappa+1
$$

for some combination of the signs, i.e.,

$$
\lambda=\frac{\left(\left(2 \kappa+1 \pm \bar{\sigma}_{3}\right)^{2}+4 \nu_{-}\right)^{2}}{16\left(2 \kappa+1 \pm \bar{\sigma}_{3}\right)^{2}} \in \mathbb{R},
$$

where $\kappa$ is some integer and $\bar{\sigma}_{3}=\sqrt{\alpha_{1}^{2}+4 \alpha_{3}} / \alpha_{1}$. 
Proposition 4.2. Real eigenvalues of the problem $(1,2)$ with $\rho(x)=1$ satisfy $\lambda \leq \sup _{x \in \mathbb{R}} \nu(x)$.

Proof. A similar result was given for $\mu(x) \equiv 0$ in Lemma 5.3 of [23]. Suppose that there is an eigenvalue $\lambda>\sup _{x \in \mathbb{R}} \nu(x)$. We can assume without loss of generality that the associated eigenfunction $\psi(x)$ has a positive maximum at some point $x=x_{0} \in \mathbb{R}$ since we may take $-\psi(x)$ if not. Hence, $\psi^{\prime \prime}\left(x_{0}\right)<0, \psi^{\prime}\left(x_{0}\right)=0$ and $\left(\lambda-\nu\left(x_{0}\right)\right) \psi\left(x_{0}\right)>$ 0 , so that $\psi(x)$ does not satisfy (1) at $x=x_{0}$. This is a contradiction. $\square$

Note that if $\lambda \leq \max \left(\nu_{-}, 0\right)$, then condition (iv) or (v) of Remark 3.5 holds. Using Theorem 1.2 and Proposition 4.2, we prove the following.

THEOREM 4.3. If the boundary value problem (1,2) with (16) has a nonzero solution, then condition (22) holds and $\max \left(\nu_{-}, 0\right)<\lambda<\alpha_{2}^{2} / 4 \alpha_{3}$.

Based on Theorem 4.3, we compute eigenvalues and eigenfunctions. For $\alpha_{1} \neq 1$, we first transform (19) by

$$
\zeta=\frac{\left(1-z_{0}\right) z}{z-z_{0}} \quad\left(z=\frac{z_{0} \zeta}{\zeta+z_{0}-1}\right)
$$

to have regular singularities at $\zeta=0,1, \infty$. We take $\zeta=z$ for $\alpha_{1}=1$.

Suppose that

$$
2 \sqrt{\lambda-\nu_{-}}+2 \sqrt{\lambda}=2 \kappa+1+\bar{\sigma}_{3}>0, \quad \kappa \in \mathbb{Z} .
$$

Then equation (22) holds with the positive sign. We set

$$
\eta(\zeta)=\zeta^{-\sigma_{1}^{+}}(\zeta-1)^{-\sigma_{2}^{+}} \psi(\zeta)
$$

so that the Riemann $P$ function (20) becomes

$$
\zeta^{-\sigma_{1}^{+}}(\zeta-1)^{-\sigma_{2}^{+}} P\left\{\begin{array}{ccc}
0 & 1 & \infty \\
\sigma_{1}^{+} & \sigma_{2}^{+} & \sigma_{3}^{+} \\
\sigma_{1}^{-} & \sigma_{2}^{-} & \sigma_{3}^{-}
\end{array}\right\}=P\left\{\begin{array}{cccc}
0 & 1 & \infty \\
0 & 0 & \kappa+1+\bar{\sigma}_{3} & \zeta \\
2 \sigma_{1}^{-} & 2 \sigma_{2}^{-} & \kappa+1
\end{array}\right\} .
$$

Hence, we obtain the hypergeometric equation

$$
\zeta(1-\zeta) \frac{\mathrm{d}^{2} \eta}{\mathrm{d} \zeta^{2}}+(c-(a+b+1) z) \frac{\mathrm{d} \eta}{\mathrm{d} \zeta}-a b \eta=0
$$

where $a=\kappa+1+\bar{\sigma}_{3}, b=\kappa+1$ and $c=1-2 \sigma_{1}^{-}=1+2 \sqrt{\lambda-\nu_{-}}$. Thus, if $\kappa$ is a negative integer, then there exists a bounded solution in (19) as

$$
\psi(\zeta)=\zeta^{\sqrt{\lambda-\nu_{-}}}(1-\zeta)^{\sqrt{\lambda}} F\left(\kappa+1+\bar{\sigma}_{3}, \kappa+1,1+2 \sqrt{\lambda-\nu_{-}} ; \zeta\right),
$$

where $F(a, b, c ; \zeta)$ is the hypergeometric function

$$
F(a, b, c ; \zeta)=\sum_{j=0}^{\infty} \frac{a(a+1) \cdots(a+j-1) b(b+1) \cdots(b+j-1)}{j ! c(c+1) \cdots(c+j-1)} \zeta^{j},
$$

which becomes a finite series when $a$ or $b$ is a nonpositive integer. For the other cases of (21), similar computations show that there is no bounded solution in (19). Thus, we have the following result. 


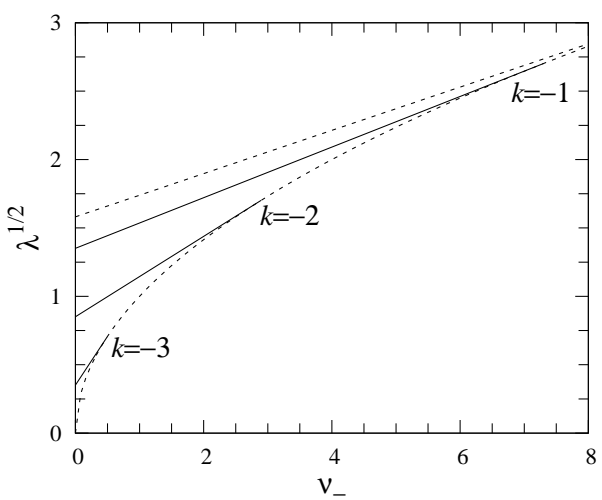

FIG. 3. Eigenvalues for (16) with $\alpha_{1}=1$ and $\alpha_{3}=10$. The dotted lines represent the upper bound $\sqrt{\lambda}=\frac{1}{2} \alpha_{2} / \sqrt{\alpha_{3}}=\frac{1}{2}\left(\alpha_{1} \nu_{-} / \sqrt{\alpha_{3}}+\sqrt{\alpha_{3}} / \alpha_{1}\right)$ and the lower bound $\sqrt{\lambda}=\sqrt{\nu_{-}}$.

THEOREM 4.4. If for some integer $\kappa \in\left(-\frac{1}{2}\left(\bar{\sigma}_{3}+1\right), 0\right)$

$$
\lambda=\frac{\left(\left(2 \kappa+1+\bar{\sigma}_{3}\right)^{2}+4 \nu_{-}\right)^{2}}{16\left(2 \kappa+1+\bar{\sigma}_{3}\right)^{2}} \in\left(\max \left(\nu_{-}, 0\right), \frac{\alpha_{2}^{2}}{4 \alpha_{3}}\right),
$$

then the boundary value problem $(1,2)$ with (16) has a nonzero solution given by $(24)$ with $\zeta=\left(1-z_{0}\right) \gamma(x) /\left(\gamma(x)-z_{0}\right)$ for $\alpha_{1} \neq 1$ and $\zeta=\gamma(x)$ for $\alpha_{1}=1$.

Eigenvalues and eigenfunctions for (16) with $\alpha_{1}=1$ and $\alpha_{3}=10$ are plotted in Figs. 3 and 4, respectively. Eigenfunctions on the first, second and third branches in Fig. 3 are given in Figs. 4(a,d), (b,e) and (c,f), respectively. Note that the hypothesis of Theorem 4.4 holds only for $\kappa=-1,-2,-3$ since $\bar{\sigma}_{3}=\sqrt{\alpha_{1}^{2}+4 \alpha_{3}} / \alpha_{1}=\sqrt{41}=$ $6.4 \ldots$

\subsection{Linear stability equation for a front in the Allen-Cahn equation.} We next consider a case in which

$$
\mu(x)=\sqrt{2}\left(\frac{1}{2}-\alpha\right), \quad \nu(x)=-3 \phi^{2}(x)+2(\alpha+1) \phi(x)-\alpha, \quad \rho(x)=1,
$$

where $\alpha$ is a constant such that $0<\alpha<1$ and

$$
\phi(x)=\frac{1}{e^{x / \sqrt{2}}+1} .
$$

For $(25)$ the eigenvalue problem $(1,2)$ is related to spectral stability of a traveling front solution with the velocity $c=\sqrt{2}\left(\frac{1}{2}-\alpha\right)$,

$$
u(t, x)=\phi(x-c t),
$$

in a PDE called the Allen-Cahn (or Nagumo) equation

$$
\frac{\partial u}{\partial t}=\frac{\partial^{2} u}{\partial x^{2}}+u(1-u)(u-\alpha)
$$

Asymptotic stability of traveling front solutions in such PDEs was studied in $[7,10,11]$ without solving the associated eigenvalue problem. Essentially the same eigenvalue problem as (25) was also considered in [23, 24]. 

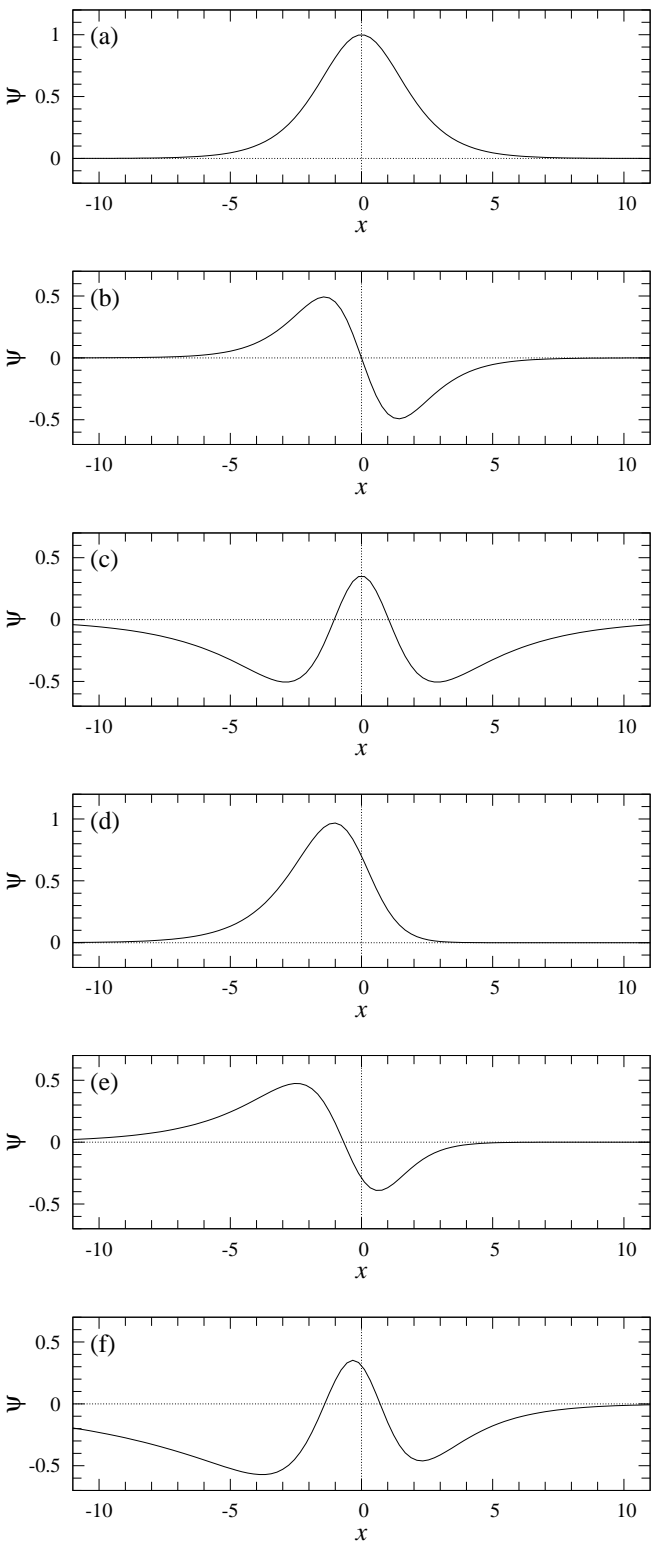

FIG. 4. Eigenfunctions for (16) with $\alpha_{1}=1$ and $\alpha_{3}=10$ : (a) $\left(\nu_{-}, \sqrt{\lambda}\right)=(0,1.35078)$; (b) $(0,0.850781)$; (c) $(0,0.350781)$; (d) $(3.5,1.99855)$; (e) $(1.5,1.29155)$; (f) $(0.25,0.528955)$.

We take $f(z)=z(1-z) / \sqrt{2}$ so that equation (3) also has a heteroclinic orbit

$$
\gamma(x)=\frac{e^{x / \sqrt{2}}}{e^{x / \sqrt{2}}+1}
$$

from $z=0$ to $z=1$. We easily see that assumptions (A1) and (A2) hold with $z_{-}=0$, $z_{+}=1$ and

$$
g(z)=\sqrt{2}\left(\frac{1}{2}-\alpha\right), \quad h(z)=-3 z^{2}+2(2-\alpha) z+\alpha-1, \quad k(z)=1 .
$$




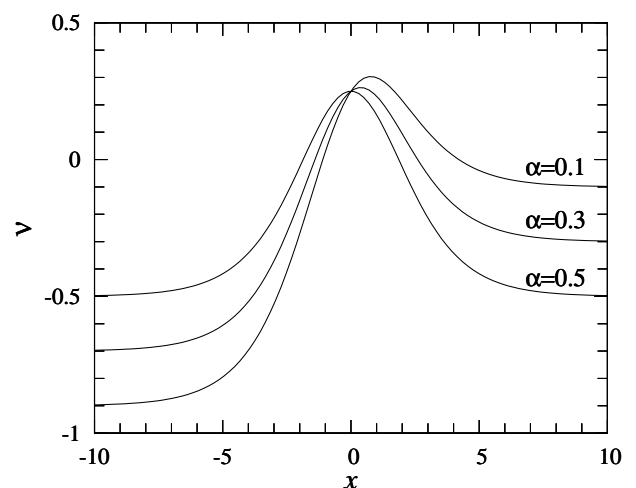

FIG. 5. Shape of the function $\nu(x)$ in (25) for $\alpha=0.1,0.3,0.5$. Note that the corresponding functions for $\alpha$ and $1-\alpha$ are symmetric about $x=0$.

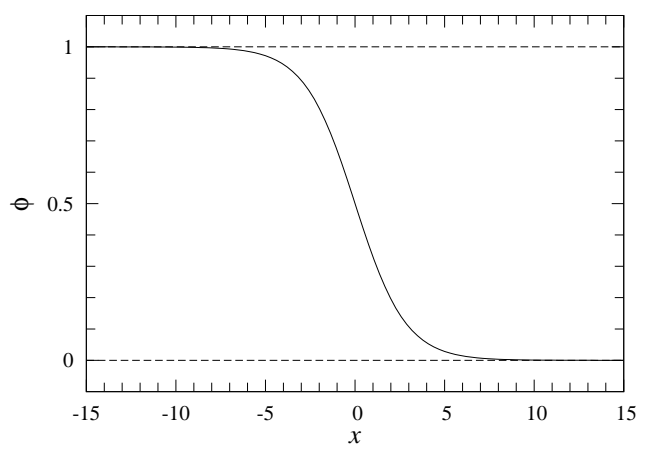

FIG. 6. Front solution (26) in the Allen-Cahn equation (27).

Condition (i) in Theorem 1.2 holds for $\alpha=\frac{1}{2}$ but does not for $\alpha \neq \frac{1}{2}$ since

$$
\mu_{ \pm}=\sqrt{2}\left(\frac{1}{2}-\alpha\right), \quad \nu_{-}=\alpha-1, \quad \nu_{+}=-\alpha, \quad \rho_{ \pm}=1,
$$

so that $\mu_{ \pm}>0$ for $\alpha \in\left(0, \frac{1}{2}\right)$ and $\mu_{ \pm}<0$ for $\alpha \in\left(\frac{1}{2}, 1\right)$. We also have

$$
\sup _{x \in \mathbb{R}} \nu(x)=\frac{1}{3}\left(\alpha^{2}-\alpha+1\right)>0
$$

for any $\alpha \in(0,1)$. See Figs. 5 and 6 for the shapes of the function $\nu(x)$ with $\alpha=$ $0.1,0.3,0.5$ and the front solution $\phi(x)$.

Equation (4) becomes

$$
\psi^{\prime \prime}+\frac{2(z+\alpha-1)}{z(z-1)} \psi^{\prime}+\frac{2(h(z)-\lambda)}{z^{2}(z-1)^{2}} \psi=0,
$$

which has only regular singularities at $z=0,1, \infty$. Solutions of (28) are expressed by a Riemann $P$ function as (20) with $z_{0}=\infty$ and

$$
\begin{aligned}
& \sigma_{1}^{ \pm}=\frac{1}{2}\left(2 \alpha-1 \pm \sqrt{8 \lambda+(2 \alpha-3)^{2}}\right) \\
& \sigma_{2}^{ \pm}=\frac{1}{2}\left(1-2 \alpha \pm \sqrt{8 \lambda+(2 \alpha+1)^{2}}\right), \quad \sigma_{3}^{+}=3, \quad \sigma_{3}^{-}=-2 .
\end{aligned}
$$




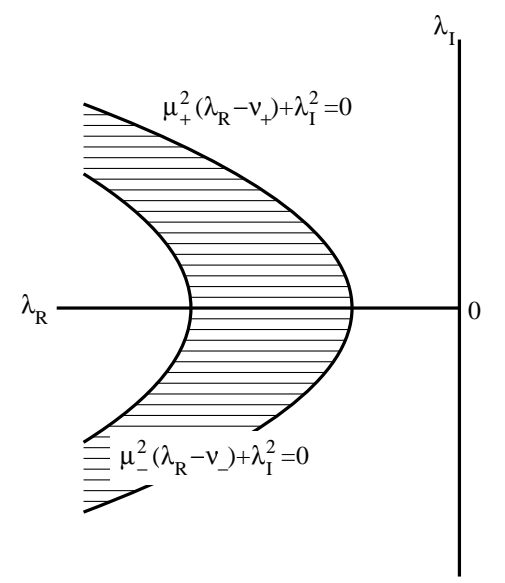

FIG. 7. Continuous spectra (the shaded region) for (25) when $\alpha \in\left(0, \frac{1}{2}\right)$. Note that $\nu_{+}>\nu_{-}$ in this case.

Using Proposition 4.1, we see that the monodromy and differential Galois groups for (28) are triangularizable if and only if

$$
\pm \sqrt{8 \lambda+(2 \alpha-3)^{2}} \pm \sqrt{8 \lambda+(2 \alpha+1)^{2}}=2 \kappa
$$

for some combination of the signs, i.e.,

$$
\lambda=\frac{\left(\kappa^{2}-4\right)(\kappa+1-2 \alpha)(\kappa-1+2 \alpha)}{8 \kappa^{2}} \in \mathbb{R}
$$

where $\kappa$ is some integer. By Theorem 1.2, Remark 3.5 and Proposition 4.2, we obtain the following result.

THEOREM 4.5. If the boundary value problem (1,2) with (25) has a nonzero solution, then one of the following conditions holds:

(i) Condition (30) holds with $\max (\alpha-1,-\alpha)<\lambda<\frac{1}{3}\left(\alpha^{2}-\alpha+1\right)$;

(ii) $\alpha \in\left(0, \frac{1}{2}\right)$ and conditions (5-) and (14) hold;

(iii) $\alpha \in\left(\frac{1}{2}, 1\right)$ and conditions $\left(5_{+}\right)$and $(15)$ hold.

Moreover, if condition (ii) or (iii) holds, then the boundary value problem $(1,2)$ with (25) has a nonzero solution.

See Fig. 7 for continuous spectra detected in Theorem 4.5(ii) for $\alpha \in\left(0, \frac{1}{2}\right)$. A similar picture can be drawn for $\alpha \in\left(\frac{1}{2}, 1\right)$. Such continuous spectra were also discussed in [23].

Based on Theorem 4.5(i), we compute discrete eigenvalues and eigenfunctions. Suppose that

$$
\sqrt{8 \lambda+(2 \alpha-3)^{2}}+\sqrt{8 \lambda+(2 \alpha+1)^{2}}=2 \kappa>0, \quad \kappa \in \mathbb{Z}
$$

We set

$$
\eta(z)=z^{-\sigma_{1}^{+}}(z-1)^{-\sigma_{2}^{+}} \psi(z),
$$


so that the Riemann $P$ function (20) becomes

$$
\begin{aligned}
& z^{-\sigma_{1}^{+}}(z-1)^{-\sigma_{2}^{+}} P\left\{\begin{array}{cccc}
0 & 1 & \infty & \\
\sigma_{1}^{+} & \sigma_{2}^{+} & 3 & z \\
\sigma_{1}^{-} & \sigma_{2}^{-} & -2 &
\end{array}\right\} \\
& =P\left\{\begin{array}{ccc}
0 & 1 & \infty \\
0 & 0 & \kappa+3 \\
-\sqrt{8 \lambda+(2 \alpha-3)^{2}} & -\sqrt{8 \lambda+(2 \alpha+1)^{2}} & \kappa-2
\end{array}\right\} .
\end{aligned}
$$

Hence, we obtain the hypergeometric equation (23) with $a=\kappa+3, b=\kappa-2$ and $c=1+\sqrt{8 \lambda+(2 \alpha-3)^{2}}$. Thus, if $\kappa=1,2$, then there exists a bounded solution in (28) as

$$
\psi(z)=z^{\sigma_{1}^{+}}(1-z)^{\sigma_{2}^{+}} F\left(\kappa+3, \kappa-2,1+\sqrt{8 \lambda+(2 \alpha-3)^{2}} ; z\right) .
$$

For the other cases of (29), similar computations show that there is no bounded solution in (28). Noting that equation (30) is not positive for $\kappa=1,2$, we prove the following result.

THEOREM 4.6. If $\lambda=0$ and

$$
\lambda=\frac{3}{2} \alpha(\alpha-1), \quad \alpha \in\left(\frac{1}{3}, \frac{2}{3}\right)
$$

respectively, then the boundary value problem $(1,2)$ with (25) has nonzero solutions given by

$$
\psi(x)=\frac{e^{x / \sqrt{2}}}{\left(e^{x / \sqrt{2}}+1\right)^{2}}
$$

and

$$
\psi(x)=\frac{e^{(1-\alpha) x / \sqrt{2}}}{e^{x / \sqrt{2}}+1}\left(1-\frac{1}{1-\alpha} \frac{e^{x / \sqrt{2}}}{e^{x / \sqrt{2}}+1}\right) .
$$

Proof. When $\kappa=1$, we have

$$
\lambda=\frac{3}{2} \alpha(\alpha-1)
$$

by $(30)$, and $\alpha \in\left(\frac{1}{3}, \frac{2}{3}\right)$ since $\lambda>\max (\alpha-1,-\alpha)$. Hence, we obtain

$$
\sigma_{1}^{+}=1-\alpha, \quad \sigma_{2}^{+}=\alpha, \quad c=4-4 \alpha,
$$

and write (31) as

$$
\psi(z)=z^{1-\alpha}(1-z)^{\alpha}\left(1-\frac{z}{1-\alpha}\right),
$$

which yields (33) by $z=\gamma(x)$. On the other hand, when $\kappa=2$, we have $\lambda=0$ and $\sigma_{1}^{+}=\sigma_{2}^{+}=1$, so that equation (31) becomes

$$
\psi(z)=z(1-z)
$$

which yields $(32)$. 


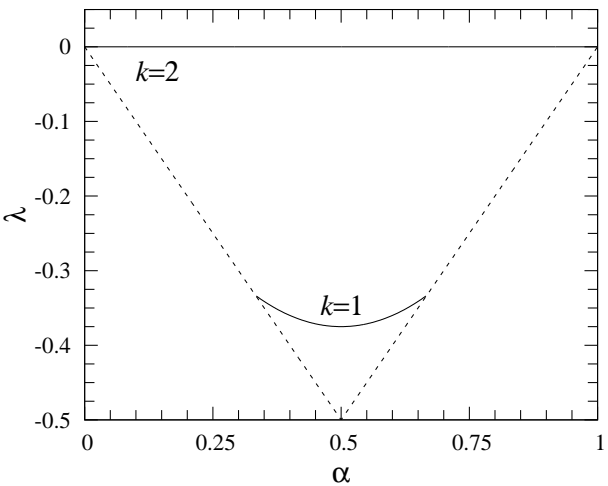

FIG. 8. Discrete eigenvalues given by (30) for $k=1,2$ in the case of (25). The dotted line represents the lower bound $\lambda=\max (\alpha-1,-\alpha)$.
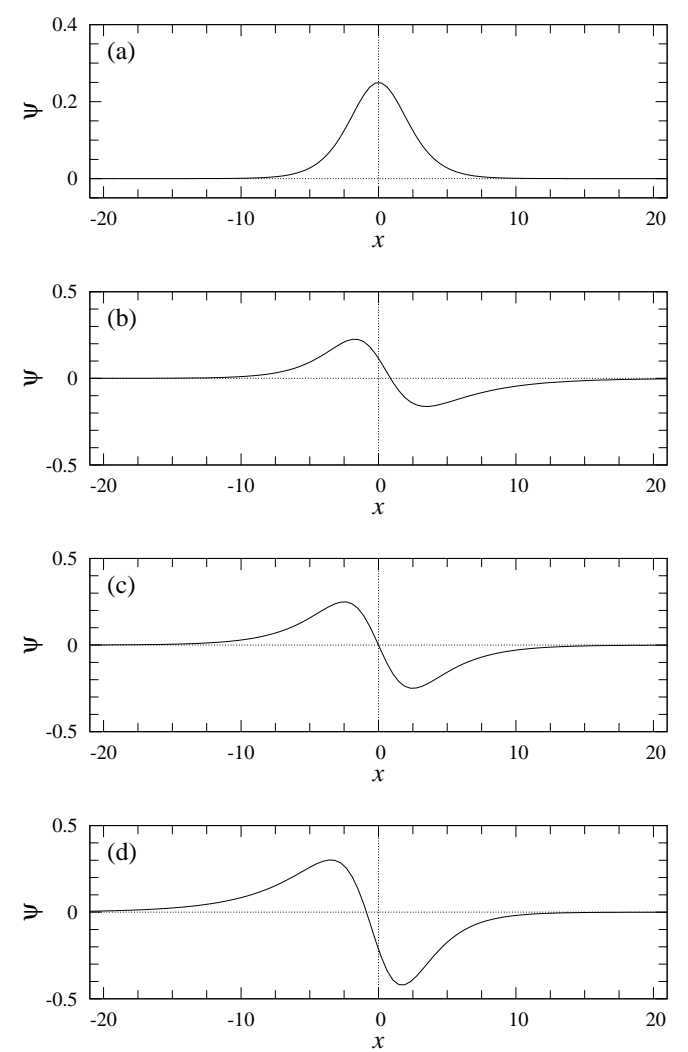

FIG. 9. Eigenfunctions for (25): (a) $\lambda=0$; (b) $\alpha=0.35$; (c) 0.5; (d) 0.65. Plates (b)-(c) show the functions for $\lambda=\frac{3}{2} \alpha(\alpha-1)$.

REMARK 4.7. The eigenfunction (32) for $\lambda=0$ can be written as

$$
\psi(x)=-\sqrt{2} \frac{\mathrm{d} \phi}{\mathrm{d} x}(x) .
$$

The existence of this eigenfunction is also guaranteed by the invariance of the PDE 

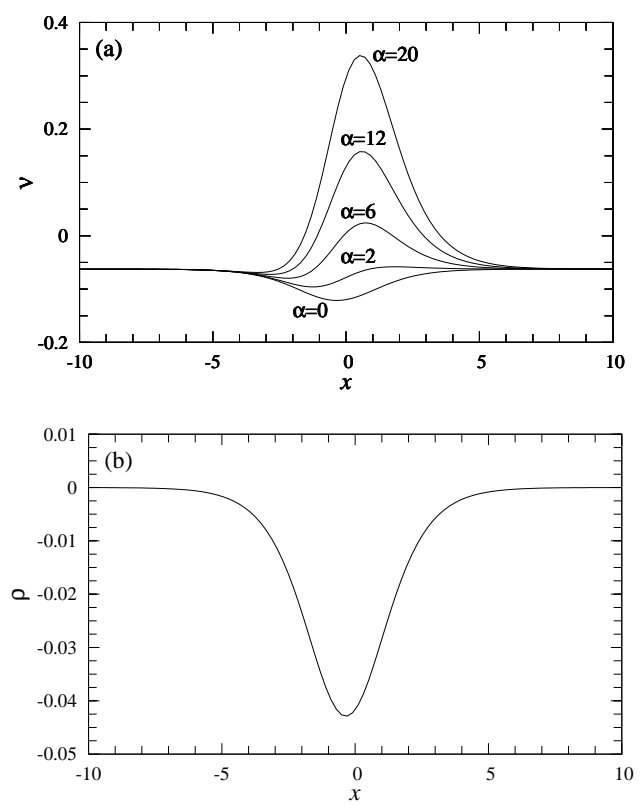

FIG. 10. Shapes of the functions $\nu(x)$ and $\rho(x)$ in (34) for $\alpha=0,2,6,12,20$.

(27) under the group of translations $x \mapsto x+x_{0}, x_{0} \in \mathbb{R}$.

Discrete eigenvalues and eigenfunctions for (25) are plotted in Figs. 8 and 9, respectively. Note that there exist continuous real spectra between $\max (\alpha-1,-\alpha)$ and $\min (\alpha-1,-\alpha)$. Nonzero discrete eigenvalues and the associated eigenfunctions in this eigenvalue problem have not been given previously, to the authors' knowledge.

4.3. Eigenvalue problem related to the Lamé equation. Finally we consider the eigenvalue problem $(1,2)$ with

$$
\begin{aligned}
& \mu(x)=0, \quad \nu(x)=\frac{\alpha e^{2 x}}{4\left(e^{x}+1\right)^{2}\left(2 e^{x}+1\right)}-\frac{4 e^{4 x}+24 e^{3 x}+28 e^{2 x}+12 e^{x}+1}{16\left(e^{x}+1\right)^{2}\left(2 e^{x}+1\right)^{2}}, \\
& \rho(x)=-\frac{e^{x}}{4\left(e^{x}+1\right)\left(2 e^{x}+1\right)} .
\end{aligned}
$$

See Fig. 10 for the shapes of $\nu(x)$ and $\rho(x)$ for $\alpha=0,2,6,12,20$. Note that $\nu(x)<0$ on $(-\infty, \infty)$ for $\alpha=0,2$. As in Sec. 4.1, we take (17) and (18) for $f(z)$ and $\gamma(x)$, so that assumptions (A1) and (A2) hold with $z_{-}=0, z_{+}=1$ and

$$
g(z)=0, \quad h(z)=\frac{\alpha z^{2}(1-z)}{4(z+1)}+\frac{3 z^{4}+2 z^{2}-8 z-1}{16(z+1)^{2}}, \quad k(z)=-\frac{z(1-z)}{4(z+1)} .
$$

We also have

$$
\mu_{ \pm}=0, \quad \nu_{ \pm}=-\frac{1}{16}, \quad \rho_{ \pm}=0
$$

Letting

$$
\varphi(z)=\left(\frac{z(1-z)}{z+1}\right)^{1 / 4} \psi(z)
$$


TABLE 1

Lamé spectral polynomial $L_{n}(\lambda)$ of (36) for $n \leq 4$. They are obtained from a general result of Table 3 in [18].

\begin{tabular}{l|l}
\hline$n$ & $L_{n}(\lambda)$ \\
\hline 0 & $\lambda$ \\
1 & $\lambda(\lambda-1)(\lambda+1)$ \\
2 & $\lambda(\lambda-3)(\lambda+3)\left(\lambda^{2}-12\right)$ \\
3 & $\lambda\left(\lambda^{2}-60\right)\left(\lambda^{2}-6 \lambda-15\right)\left(\lambda^{2}+6 \lambda-15\right)$ \\
4 & $\lambda\left(\lambda^{2}-208\right)\left(\lambda^{2}-28\right)\left(\lambda^{2}-10 \lambda-7\right)\left(\lambda^{2}+10 \lambda-7\right)$ \\
\hline
\end{tabular}

TABLE 2

Lamé functions of (36) for $1 \leq n \leq 4$. They are obtained based on general results of Tables 2 and 3 in [18].

\begin{tabular}{c|c|l}
\hline$n$ & $\lambda$ & $\varphi(z)$ \\
\hline 0 & 0 & 1 \\
\hline 1 & 0 & $\sqrt{z}$ \\
& \pm 1 & $\sqrt{z \mp 1}$ \\
\hline 2 & 0 & $\sqrt{(z-1)(z+1)}$ \\
& \pm 3 & $\sqrt{z(z \pm 1)}$ \\
& $\pm 2 \sqrt{3}$ & $z \mp 1 / \sqrt{3}$ \\
\hline 3 & 0 & $\sqrt{z(z-1)(z+1)}$ \\
& $\pm 2 \sqrt{15}$ & $(z \mp 1 / \sqrt{3}) \sqrt{z}$ \\
& $3 \pm 2 \sqrt{6}$ & $\left(z+\frac{1}{5}(1 \mp \sqrt{6})\right) \sqrt{z-1}$ \\
& $-3 \pm 2 \sqrt{6}$ & $\left(z-\frac{1}{5}(1 \pm \sqrt{6})\right) \sqrt{z+1}$ \\
\hline 4 & 0 & $z^{2}-\frac{3}{5}$ \\
& $\pm 2 \sqrt{7}$ & $(z \mp 1 / \sqrt{7}) \sqrt{(z-1)(z+1)}$ \\
& $\pm 4 \sqrt{13}$ & $z^{2} \mp \frac{2}{7} \sqrt{13} z+\frac{1}{7}$ \\
& $5 \pm 3 \sqrt{2}$ & $\left(z+\frac{1}{14}(2 \mp 3 \sqrt{2})\right) \sqrt{z(z-1)}$ \\
& $-5 \pm 3 \sqrt{2}$ & $\left(z-\frac{1}{14}(2 \pm 3 \sqrt{2})\right) \sqrt{z(z+1)}$ \\
\hline
\end{tabular}

we rewrite (4) with (35) as

$$
\chi(z) \varphi^{\prime \prime}+\frac{1}{2} \chi^{\prime}(z) \varphi^{\prime}-(\alpha z+\lambda) \varphi=0,
$$

where $\chi(z)=4\left(z^{3}-z\right)$.

Equation (36) is a special case of the Lamé equation [28], which has four regular singularities at $z=0, \pm 1, \infty$. Its monodromy group, i.e., differential Galois group, is triangularizable if and only if $\alpha=n(n+1)$ for some nonnegative $n \in \mathbb{Z}$ and $\lambda$ is a root of a $2 n+1$-th order polynomial $L_{n}(\lambda)$. See Chapter 4 of [26] for the details. The polynomial $L_{n}(\lambda)$ is called the Lamé spectral polynomial [18] and given in Table 1 for $n \leq 4$ in the special case of (36). When these conditions are satisfied, the Lamé equation (36) has a solution of the form

$$
\varphi(z)=z^{\varepsilon_{0} / 2}(z-1)^{\varepsilon_{1} / 2}(z+1)^{\varepsilon_{-1} / 2} q(z),
$$

where $\varepsilon_{j}=0$ or 1 for $j=0, \pm 1$, and $q(z)$ is a polynomial of $z$. The solution (37) is also called the Lamé function $[18,26]$ and given in Table 2 for $n \leq 4$ in our case. For higher values of $n$, the Lamé spectral polynomials and functions are very complicated. 
See Section 3 of [18] for higher-order Lamé spectral polynomials and functions with $5 \leq n \leq 8$.

Rewrite (36) as the form (6) and let $M_{0}, M_{ \pm 1}$ and $M_{\infty}$ be the monodromy matrices for small loops around the singularities $z=0, \pm 1$ and $\infty$, respectively. By a general property, the products of all monodromy matrices are the identity whatever their order is. Hence, the monodromy group of (36) is generated by $M_{0}, M_{1}$ and $M_{\infty}$, say. On the other hand, the local exponents at $z=0, \pm 1$ are 0 and $\frac{1}{2}$, so that $M_{0}$ and $M_{ \pm 1}$ have eigenvalues \pm 1 . This implies that $M_{0}^{2}=M_{ \pm 1}^{2}=\mathrm{id}$, where id is the identity matrix. Using this fact and noting that $M_{-1} M_{0} M_{\infty} M_{1}=M_{-1} M_{\infty} M_{0} M_{1}=$ id, we show that

$$
M_{0} M_{\infty}=M_{\infty} M_{0}=M_{-1} M_{1},
$$

i.e., $M_{0}$ and $M_{\infty}$ are commutative. Similarly, $M_{1}$ and $M_{\infty}$ are shown to be commutative. Based on these arguments, we prove the following lemma.

LeMma 4.8. Let $\Gamma_{\text {loc }}$ be any simply connected neighborhood of $\{x \in \mathbb{R} \mid 0 \leq x \leq 1\}$ in $\mathbb{C}$. Then the restriction of (36) on $\Gamma_{\text {loc }}$ has a triangularizable differential Galois group if and only if $\alpha=n(n+1)$ and $\lambda$ is a root of $L_{n}(\lambda)$ for some nonnegative integer $n$.

Proof. Since the sufficiency is obvious, we only prove the necessity of the conditions.

Suppose that the differential Galois group for the restriction of (36) onto $\Gamma_{\text {loc }}$ is triangularizable. Then the corresponding monodromy group, which is generated by $M_{0}$ and $M_{1}$, is also triangularizable by Theorem 2.5. Choose the fundamental matrix such that $M_{0}$ and $M_{1}$ are triangular. Then we can express $M_{0}$ and $M_{1}$ in the form

$$
\left(\begin{array}{cc} 
\pm 1 & c \\
0 & \mp 1
\end{array}\right), \quad c \in \mathbb{C},
$$

since their eigenvalues are \pm 1 . Using this expression and the fact that $M_{\infty}$ is commutative with $M_{0}$ and $M_{1}$, we easily see that $M_{\infty}$ is also triangular for this fundamental matrix. Since the corresponding monodromy group is generated by the triangular matrices $M_{0}, M_{1}$ and $M_{\infty}$, it follows from Theorem 2.5 that the differential Galois group of (36) on $\mathbb{P}^{1} \backslash\{0, \pm 1, \infty\}$ is also triangularizable. Thus, we obtain the result. $\mathbf{Q}$

Using Lemma 4.8 and applying Theorem 1.2 we have the following result.

THEOREM 4.9. If the boundary value problem $(1,2)$ with (34) has a nonzero solution, then $\alpha=n(n+1)$ and $\lambda$ is a root of $L_{n}(\lambda)$ for some nonnegative integer $n$.

When $\alpha<5 \cdot 6=30$, we use Theorem 4.9 and Table 2 to easily solve the eigenvalue problem for (34) as follows:

(i) For $\alpha=6(n=2), \lambda=-3$ and

$$
\psi(x)=(z(1-z)(z+1))^{1 / 4}=\left(\frac{e^{x}\left(1+2 e^{x}\right)}{\left(1+e^{x}\right)^{3}}\right)^{1 / 4} ;
$$

(ii) for $\alpha=12(n=3), \lambda=0$ and

$$
\psi(x)=\left(z(1-z)(z+1)^{3}\right)^{1 / 4}=\left(\frac{e^{x}\left(1+2 e^{x}\right)^{3}}{\left(1+e^{x}\right)^{5}}\right)^{1 / 4} ;
$$



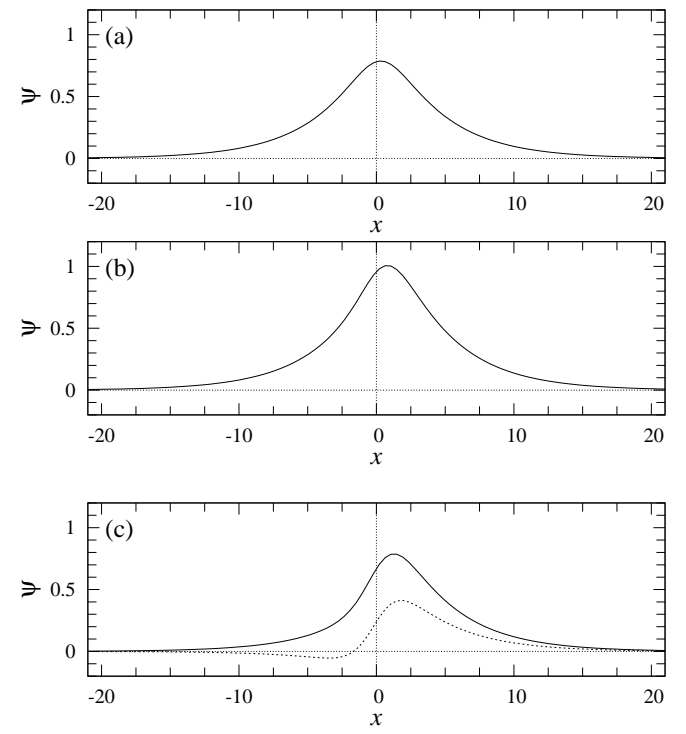

FIG. 11. Eigenfunctions for (34): (a) $\alpha=6$ and $\lambda=-3$; (b) $\alpha=12$ and $\lambda=0$; (c) $\alpha=20$ and $\lambda=5 \pm 3 \sqrt{2}$. In plate (c), the solid and dotted lines represent eigenfunctions for $\lambda=5-3 \sqrt{2}$ and $\lambda=5+3 \sqrt{2}$, respectively.

(iii) for $\alpha=20(n=4), \lambda=5 \pm 3 \sqrt{2}$ and

$$
\begin{aligned}
\psi(x) & =\left(z+\frac{1}{14}(2 \mp 3 \sqrt{2})\right)(z(1-z))^{1 / 4} \\
& =\left(\frac{e^{x}}{e^{x}+1}+\frac{1}{14}(2 \mp 3 \sqrt{2})\right)\left(\frac{e^{x}\left(1+2 e^{x}\right)}{\left(1+e^{x}\right)^{3}}\right)^{1 / 4} .
\end{aligned}
$$

For other values of $\alpha<30$, this eigenvalue problem has no solution. These eigenfunctions are plotted in Fig. 11.

Acknowledgments. This work was partially supported by the Japan Society for the Promotion of Science (JSPS) through Grant-in-Aid for JSPS Fellows No. 21.09222. DBS acknowledges support from MICINN-FEDER grant MTM2009-06973 and CURDIUE grant 2009SGR859, and thanks his colleagues at Instituto de Matemáticas y sus Aplicaciones, Universidad Sergio Arboleda, for helpful discussion and encouragement. KY appreciates support from the JSPS through Grant-in-Aid for Scientific Research (C) Nos. 21540124 and 22540180.

\section{REFERENCES}

[1] P. B. Acosta-Humánez, Galoisian Approach to Supersymmetric Quantum Mechanics, Ph.D. thesis, Universitat Politècnica de Catalunya, 2009.

[2] P. B. Acosta-Humánez, J. J. Morales-Ruiz, and J.-A. Weil, Galoisian approach to integrability of Schrödinger equation, Rep. Math. Phys., to appear.

[3] S. Allen And J. W. Cahn, A microscopic theory for antiphase boundary motion and its application to antiphase domain coarsening, Acta Metall., 27 (1979), pp. 1084-1095.

[4] W. O. Amrein, A. M. Hinz, And D. B. Pearson (eds.), Sturm-Liouville Theory: Past and Present, Birkhäuser, Basel, 2005.

[5] D. Blázquez-Sanz and J. J. Morales-Ruiz, Differential Galois theory of algebraic Lie-Vessiot systems, in: P. B. Acosta-Humánez and F. Marcellán (eds.), Differential Algebra, Complex 
Analysis and Orthogonal Polynomials, Contemp. Math., Vol. 509, American Mathematical Society, Providence, RI, 2010, pp. 1-58.

[6] D. Blázquez-Sanz and K. Yagasaki, Analytic and algebraic conditions for bifurcations of homoclinic orbits I: Saddle equilibria, J. Differential Equations, 253 (2012), pp. 2916-2950.

[7] X. Chen, Existence, uniqueness, and asymptotic stability of traveling waves in nonlocal evolution equations, Adv. Differential Equations, 2 (1997), pp. 125-160.

[8] E. A. Coddington and N. Levinson, Theory of Ordinary Differential Equations, McGrawHill, New York, 1955.

[9] F. Fauvet, J.-P. Ramis, F. Richard-Jung, and J. Thomann, Stokes phenomenon for the prolate spheroidal wave equation, Appl. Numer. Math., 60 (2010), pp. 1309-1319.

[10] P. C. Fife And J. B. MCLEOD, The approach of solutions of nonlinear diffusion equations to travelling front solutions, Arch. Ration. Mech. Anal., 65 (1977), pp. 335-361.

[11] P. C. Fife AND J. B. MCLEOD, A phase plane discussion of convergence to travelling fronts for nonlinear diffusion, Arch. Rational Mech. Anal., 75 (1981), pp. 281-314.

[12] L. HulthéN, Über die Eigenlösungen der Schrödinger-Gleichung des Deuterons, Ark. Mat. Astr. Fys., 28A (1942), no. 5.

[13] E. L. Ince, Ordinary Differential Equations, Dover Publications, New York, 1956.

[14] I. Kaplansy, Introduction to Differential Algebra, Hermann, Paris, 1957.

[15] H. Kleinert, Path Integrals in Quantum Mechanics, Statistics, Polymer Physics, and Financial Markets, 3rd ed., World Scientific, Singapore, 2004.

[16] T. KimuRA, On Riemann's equations which are solvable by quadratures, Funkcial. Ekvac., 12 (1969), pp. 269-281.

[17] L. D. Landau and E. M. Lifshitz, Quantum Mechanics Non-Relativistic Theory, Course of Theoretical Physics, Vol. 3, Addison-Wesley, Reading, Mass., 1958.

[18] R. S. Maier, Lamé polynomials, hyperelliptic reductions and Lamé band structure, Philos. Trans. R. Soc. Lond. Ser. A, 366 (2008), pp. 1115-1153.

[19] J. J. Morales-Ruiz, Differential Galois Theory and Non-Integrability of Hamiltonian Systems, Birkhaüser, Basel, 1999.

[20] J. J. Morales-Ruiz And J. M. Peris, On a Galoisian approach to the splitting of separatrices, Ann. Fac. Sci. Toulouse Math. (6), 8 (1999), pp. 125-141.

[21] J. SADEGHI, Raising and lowering of generalized Hulthén potential from supersymmetry approaches, Internat. J. Theoret. Phys., 46 (2007), pp. 492-502.

$[22]$ B. Sandstede, Stability of travelling waves, in: B. Fiedler (Ed.), Handbook of Dynamical Systems, Vol. 2, North-Holland, Amsterdam, 2002, Chapter 18, pp. 983-1055.

[23] D. H. Sattinger, On the stability of waves of nonlinear parabolic systems, Advances in Math., 22 (1976), pp. 312-355.

[24] D. H. SATtinger, Weighted norms for the stability of traveling waves, J. Differential Equations, 25 (1977), pp. 130-144.

[25] M. van Der Put and M. F. Singer, Galois Theory of Linear Differential Equations, Springer, New York, 2003.

[26] A. VAN DER WAALl, Lamé Equations with Finite Monodromy, Ph.D, thesis, Universiteit Utrecht, Utrecht, 2002. On-line reference: http: //igitur-archive.library.uu.nl/dissertations/2002-0530-113355/ UUindex.html.

[27] G. Walter and T. Soleski, A new friendly method of computing prolate spheroidal wave functions and wavelets, Appl. Comput. Harmon. Anal., 19 (2005), pp. 432-443.

[28] E. T. Whittaker and G. N. Watson, A Course of Modern Analysis, Cambridge University Press, Cambridge, 1927.

[29] K. YAGASAKI, Galoisian obstructions to integrability and Melnikov criteria for chaos in two-degree-of-freedom Hamiltonian systems with saddle centres, Nonlinearity, 16 (2003), pp. 2003-2012.

[30] A. ZettL, Sturm-Liouville Theory, American Mathematical Society, Providence, RI, 2005.

[31] M. ZNOJIL, Exact solution of the Schrodinger and Klein-Gordon equations for generalised Hulthen potentials, J. Phys. A, 14 (1981), pp. 383-394. 